استفاده از يرتوتابى كَاما براى حذف آلودَّى باكتريايى آغوز كَاو

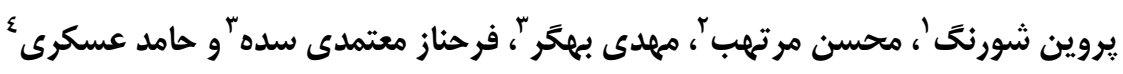

1- استاديار، يُوهشخاه علوم و فنون هستهاى، يُوهشكده كشاورزى هستهاى (نويسنده مسوول: pshawrang@ @ nrcam.org)

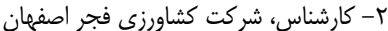

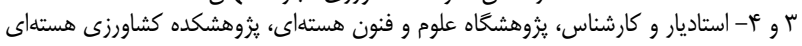

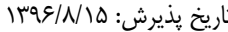

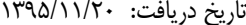

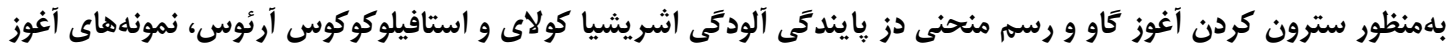

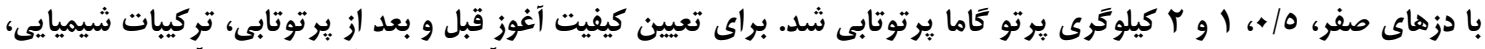

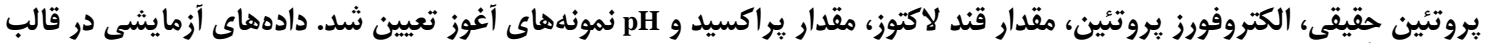

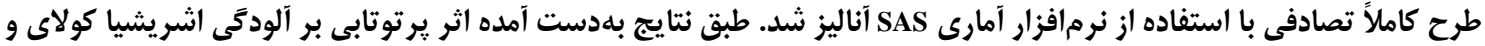

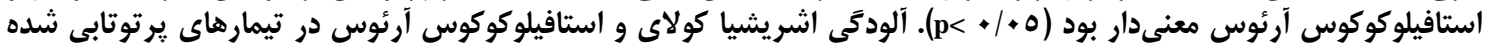

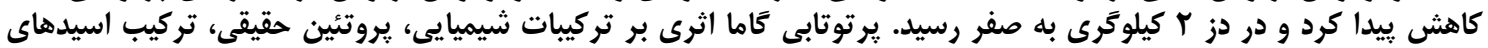

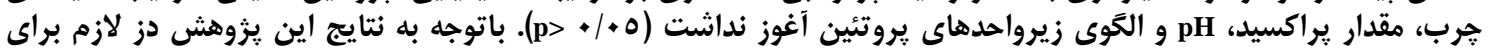

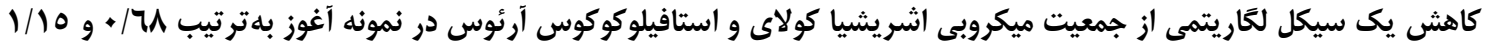

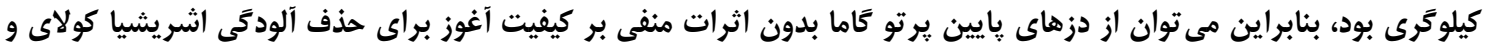
استافيلوكوكوس آرئوس استفاده كرد داين

وازههاى كليدى: آغوز، ير توتابى تاما، استافيلوكوكوس آرئوس، اشريشيا كولاي، تركيبات شيميايى

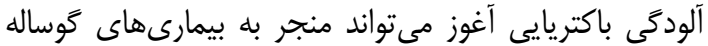

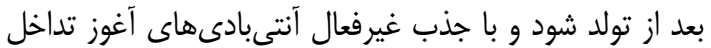

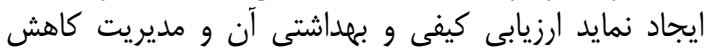

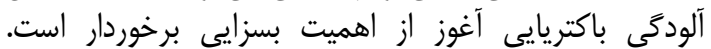

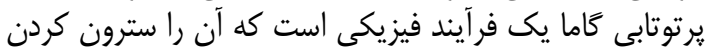

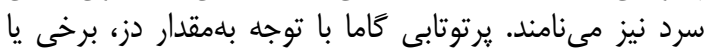

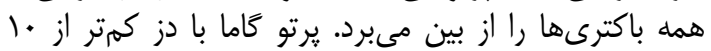

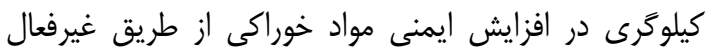

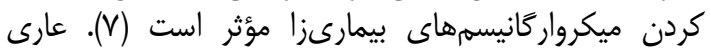

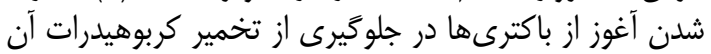

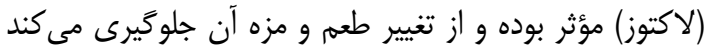

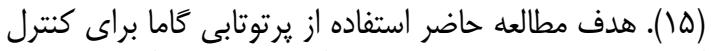

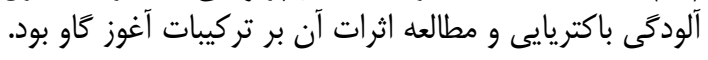

\section{مواد و روشها ير توتابى نمونههاى آغوز}

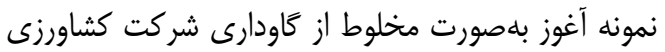

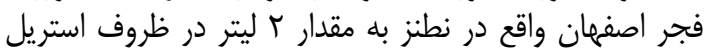

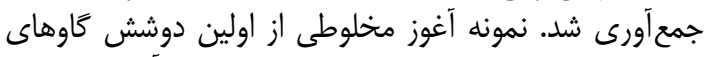

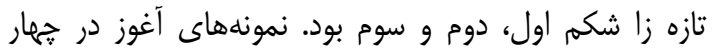

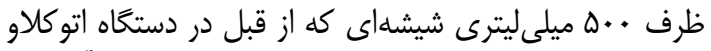

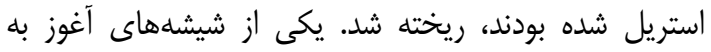

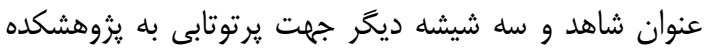

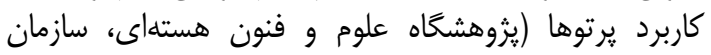

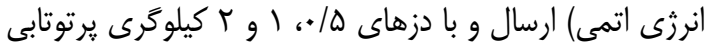

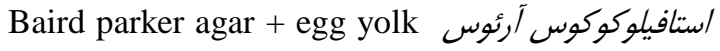

مقدمه

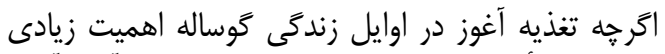

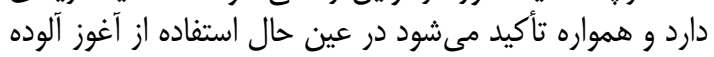

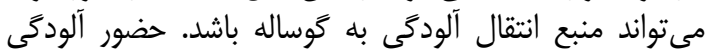

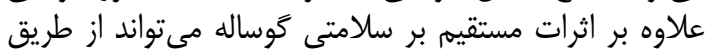

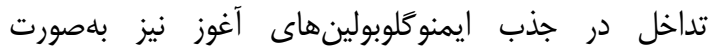

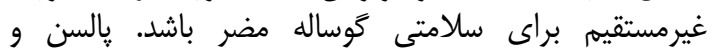

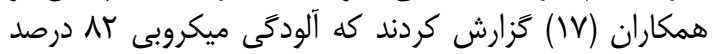

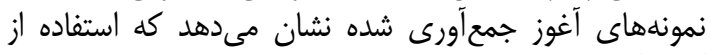

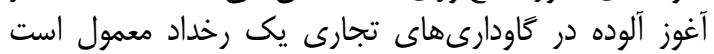

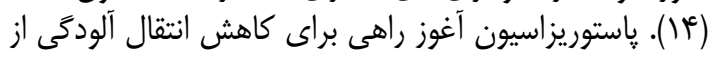

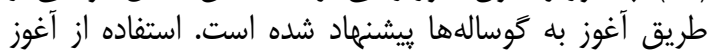

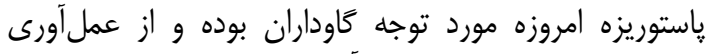
حرارتى براى ياستوريزه كردن آغوز استفاده مى كنند. تحقيقات

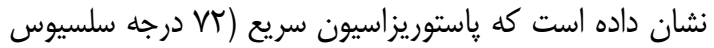

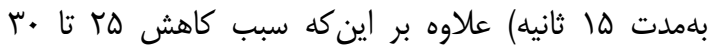
درصدى غلظت ايمونوكلوبولين G (ميلى كرم در ميلى ليليتر)

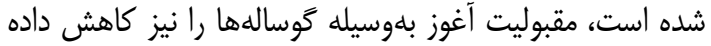

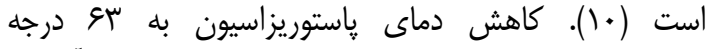

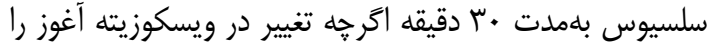

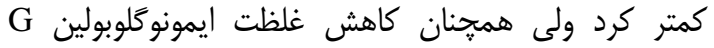

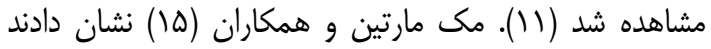

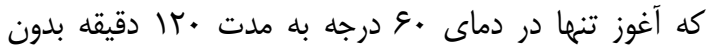

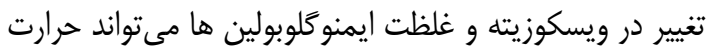

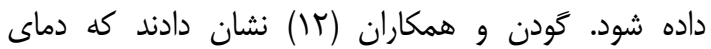

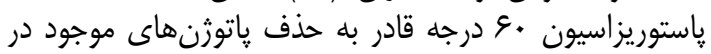

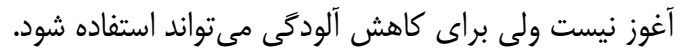

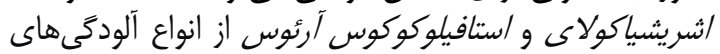

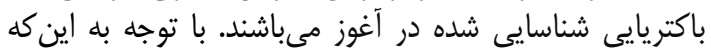




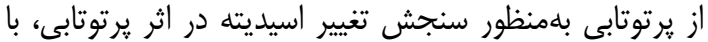

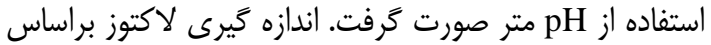

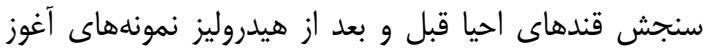

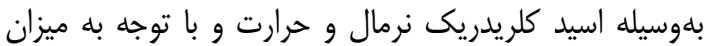

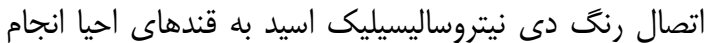

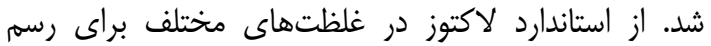

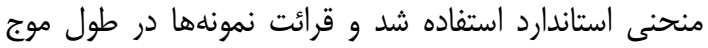
والحاى هf.

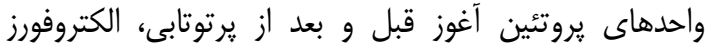

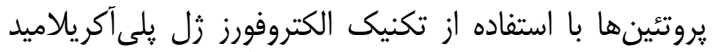

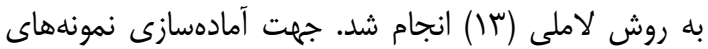

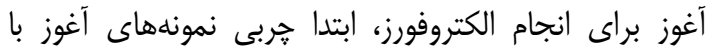

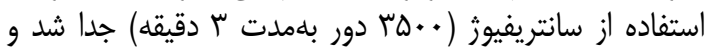

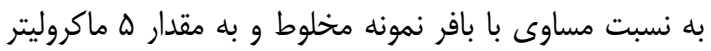

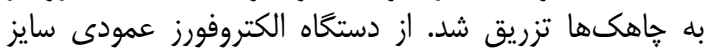
متوسط شركت اختريان براى انجام البام الكتروفورز استفاده شد.

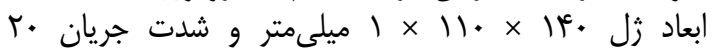

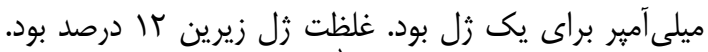

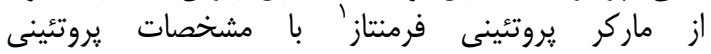

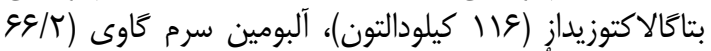

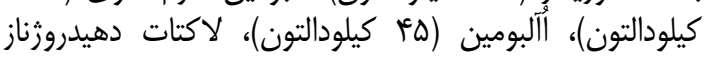

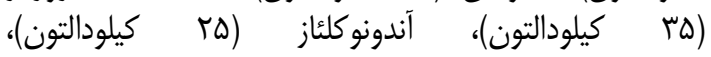

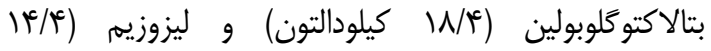

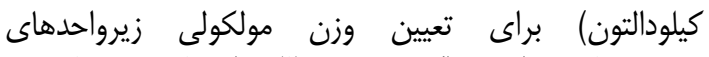
بروتئينهاى مواد خوراكى مورد مطالعه استفاده شد. با بوني توجيه

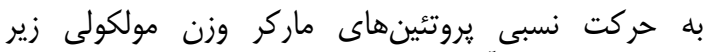
واحدهاى بروتئين أغوز تعيين شدا.

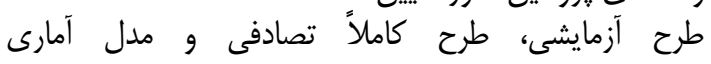
بود. در اين مدل Yij $=\mu+T i+$ Tij

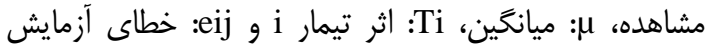

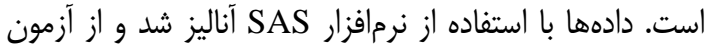

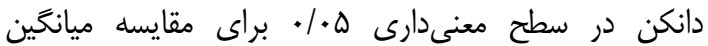
استفاده شد.

\section{نتايج و بحث}

بار ميكروبى و منحنى دز / پايندكى آلودتى إنهاى آغوز

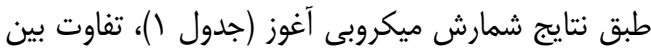

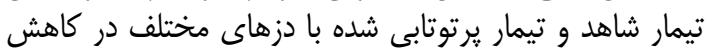

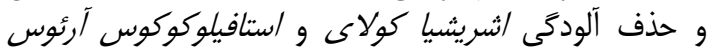

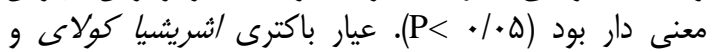

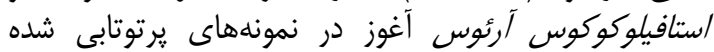

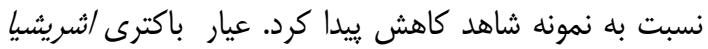

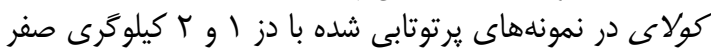

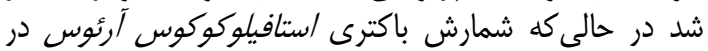

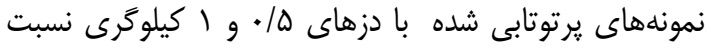

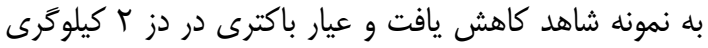

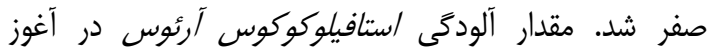

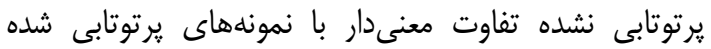

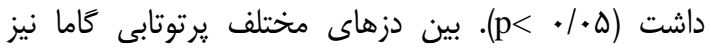

و براى و اشريشياكولاى بود. محيطهاى كشت طبق توك توصيه شركت سازنده

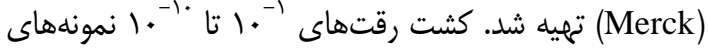

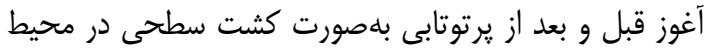

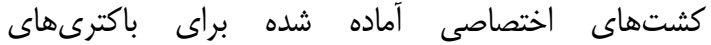

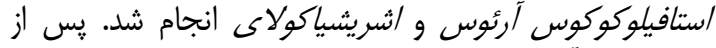

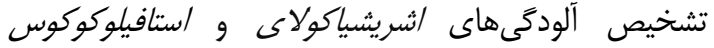

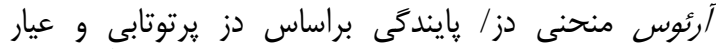

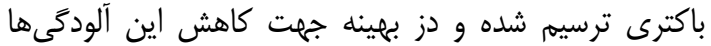

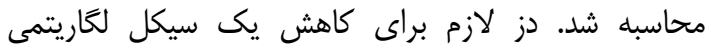
آلودخى بلوسيله معادله زير محاسبه شد:

$\mathrm{D}=\left(\mathrm{D}_{2}-\mathrm{D}_{1}\right) /\left(\log \mathrm{N}_{1}-\log \mathrm{N}_{2}\right)$

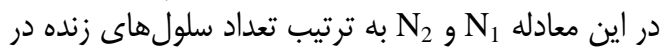

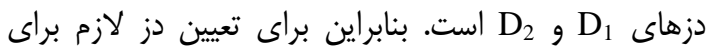

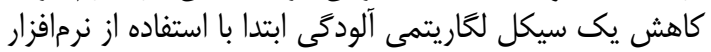

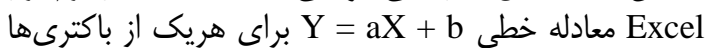

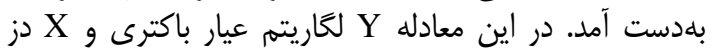

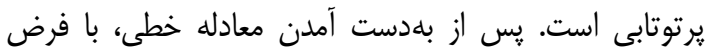

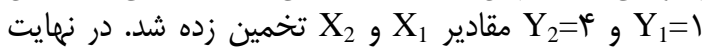

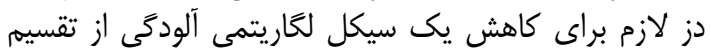

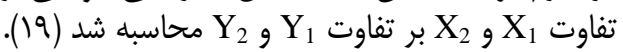
ارزيابى كيفيت آغوز براى تعيين اثرات يرتوتابى كاما بر تركيبات آغوزيت آغوز، مقدار و و

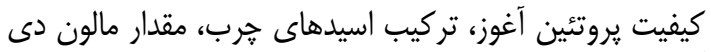

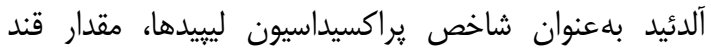

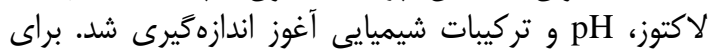

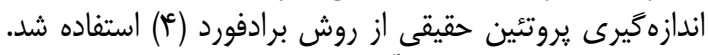

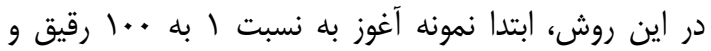

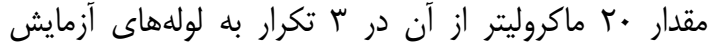

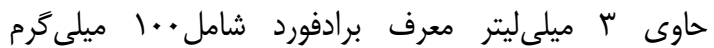

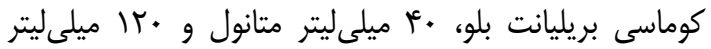

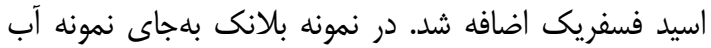

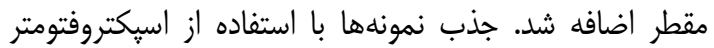

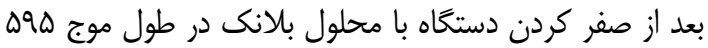

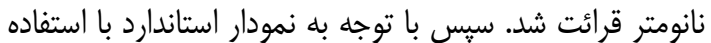

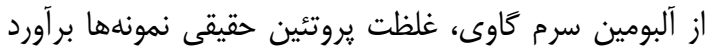

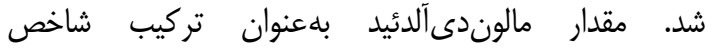

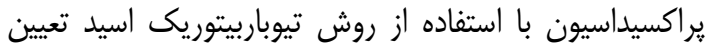

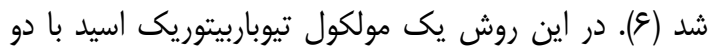

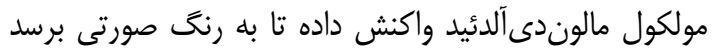

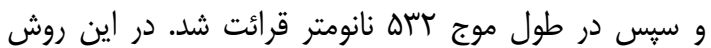

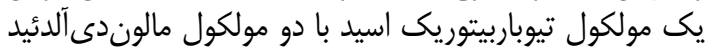

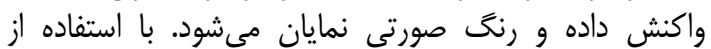

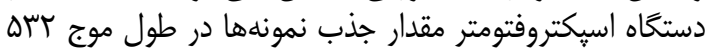

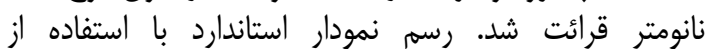

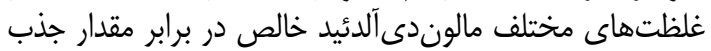

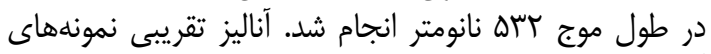

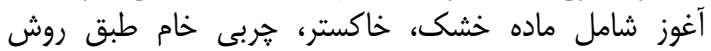

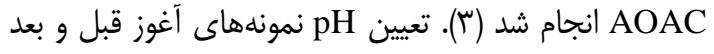


شده است (9). تحقيقات نشان داده است كه يرتوتابى كاما در

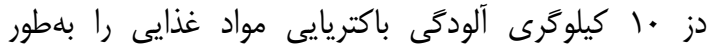

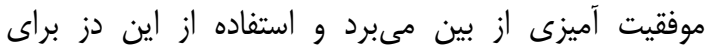

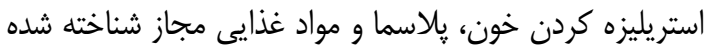

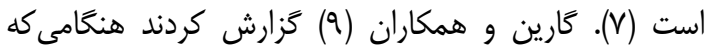
آغوزهايى كه با بروسلا آبرتوس، اشتريشياكولاى

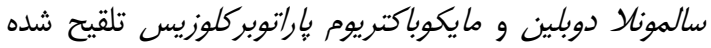

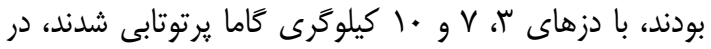

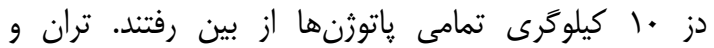

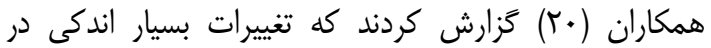

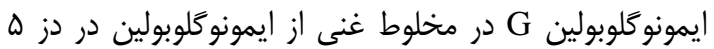

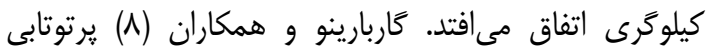

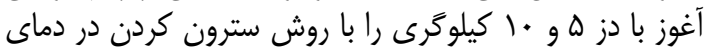

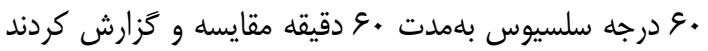

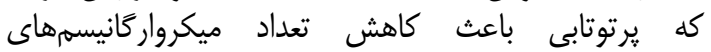

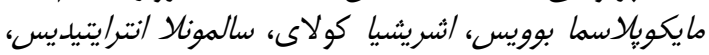

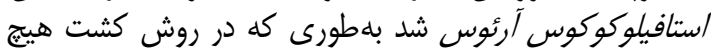
كلونى مشاهده نشد. در اين مطالعه مقدار ايمونوكلوبون إنولينها نيز

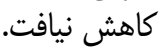

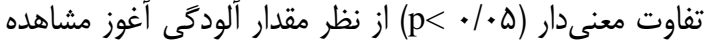

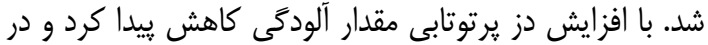

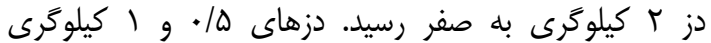

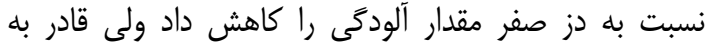

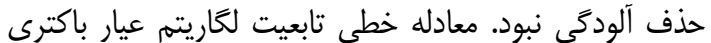

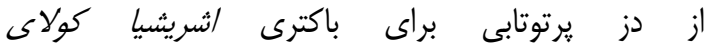

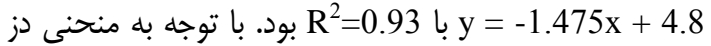

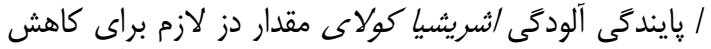

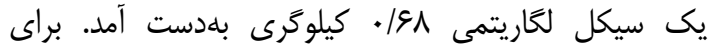
باكترى استافيلوكوكوس آرئوس معادله خطى تاكئ تابعيت لكاريته

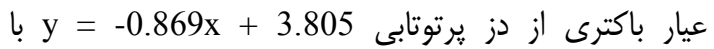

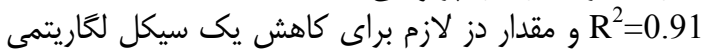

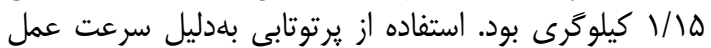

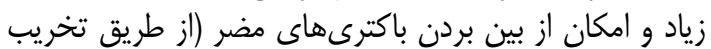

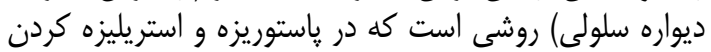

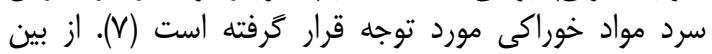

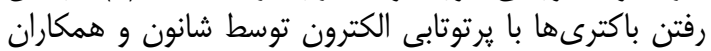

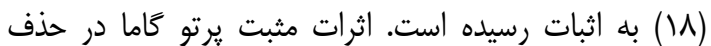

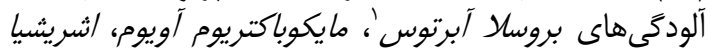

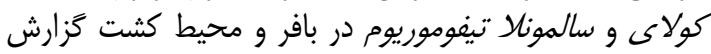

جدول ا- شمارش ميكروبى آغوز قبل و بعد از يرتوتابى (log CFU/ml) Table 1. Microbial counts of the pre-and post irradiated colostrums sample (log CFU/ml)

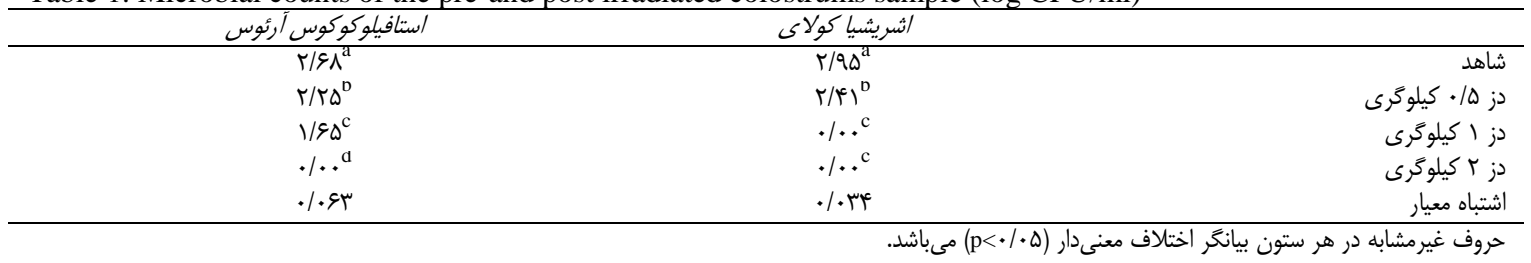

ديواره سلولى) روشى است كه در سترون كردن و استريليزه

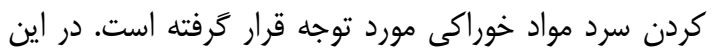

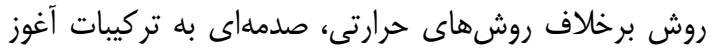

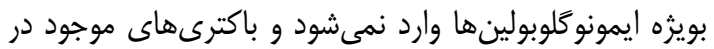

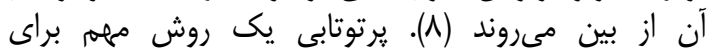

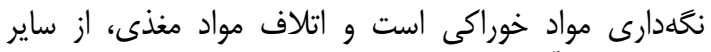
روشهاى فرآورى كمتر است (V)

\section{تركيبات شيميايى آغوز}

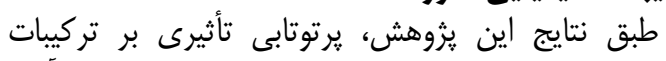

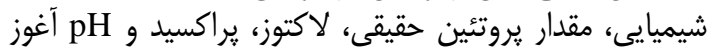

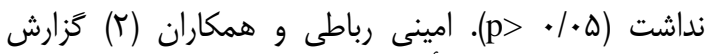

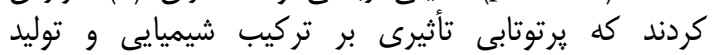

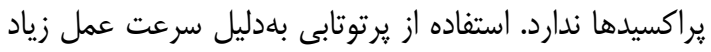

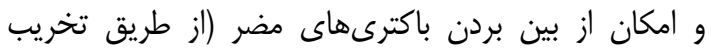

Table 2. Chemical composition of the pre-and post irradiated colostrums sample

جدول r- تركيبات شيميايى آغوز قبل و بعد از يرتوتابى

\begin{tabular}{|c|c|c|c|c|c|c|c|c|}
\hline $\mathrm{pH}$ & $\begin{array}{c}\text { يراكسيد } \\
(\mathrm{nmol} / 100 \mathrm{ml})\end{array}$ & $\begin{array}{l}\text { لاكتوز) } \\
\end{array}$ & 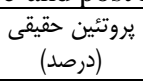 & يروتئين خام & (درصد) & خاكستر & ماده خشك (درصد & \\
\hline $8 / 4 \pi$ & P/.Tr & $T / V^{c}$ & $I V / Y Y^{c}$ & W/DF & $9 / 91^{4}$ & $r / \Delta \omega$ & $r N / \Delta V$ & شاهد \\
\hline $8 / 4)$ &.$/ .11$ & $T / A F$ & $\mid V / \& A$ & W/9D & S/QT & $T / M \Lambda$ & TN/QV & ه/·•كيلوكرى \\
\hline$g / 41$ & . /.r. & $r / 99$ & $\mid V / F$ & W/Ar & $g / V$. & ו ג & TN/DT & اكيلوَرى \\
\hline$s / 4$. &.$/ \cdot T V$ & $r / 9 \Lambda$ & $I V / 9 D$ & N/S. & $s / 4 \mid$ & $r / \Delta$. & $r q / \cdot \Lambda$ & r كيلوكرى \\
\hline$\% 1$ & $.1 . .19$ &.$/ . \mathrm{rV}$ &.$/ 11 r$ & .1 .14 & $.1 . \mathrm{Va}$ & .1 .98 & هr/I. & اشتباه معيار \\
\hline
\end{tabular}



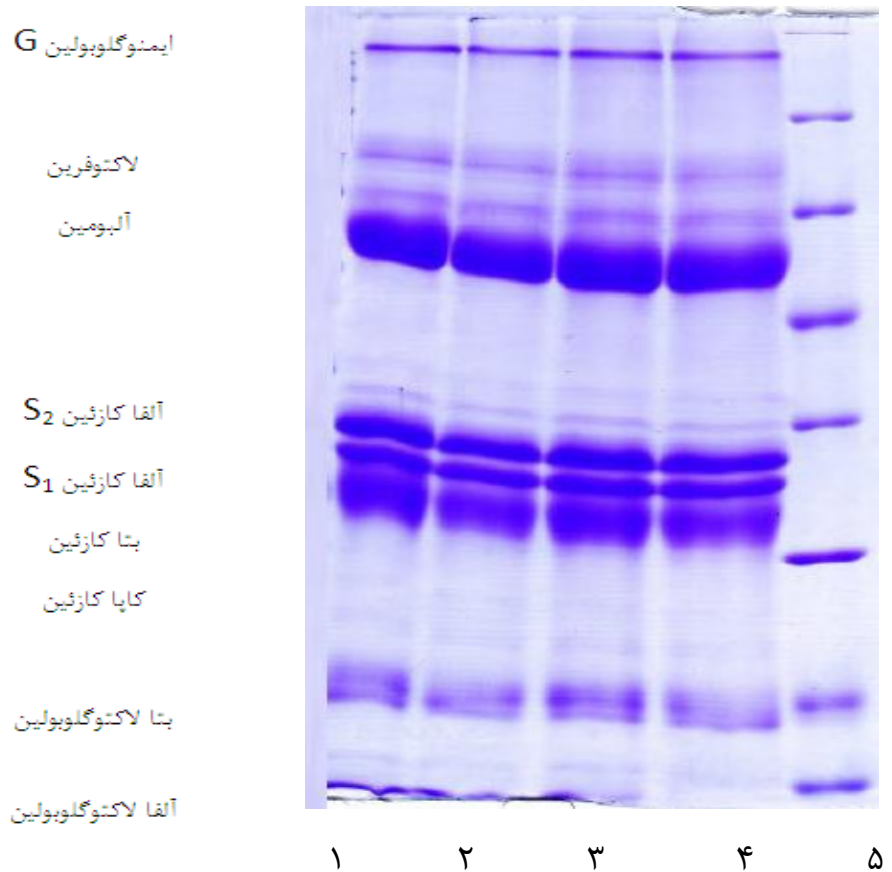

$116 \mathrm{KDa}$

$66.2 \mathrm{KDa}$

$45 \mathrm{KDa}$

$35 \mathrm{KDa}$

$25 \mathrm{KDa}$

$18.4 \mathrm{KDa}$

14.4 KDa

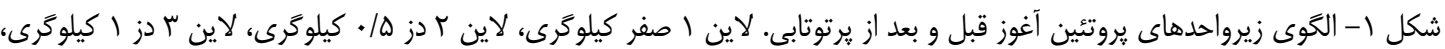

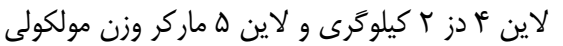

Figure 1. Electrophoretic pattern of proteins pre-and post irradiated colostrums. Line 1: 0 kGy, Line 2: 0.5 kGy, Line 3: $1 \mathrm{kGy}$, Line 4: $2 \mathrm{kGy}$, Line 5: Molecular-weight size marker

كزارش شده است. طبق نتايج الكتروفورز زل يلى آكريلاميد

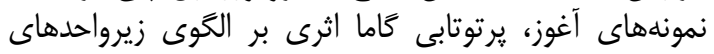

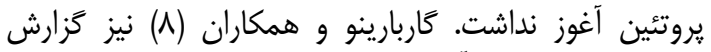

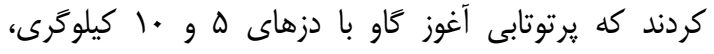

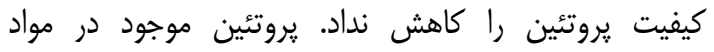

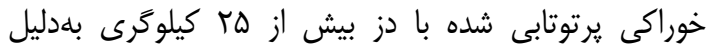

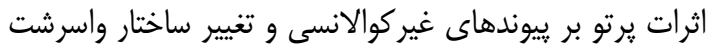

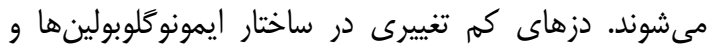

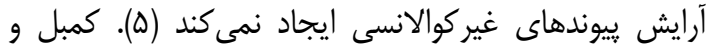

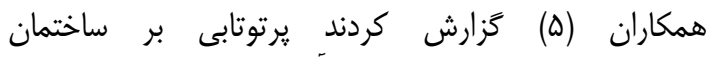

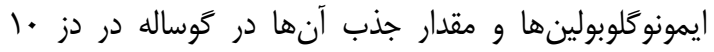

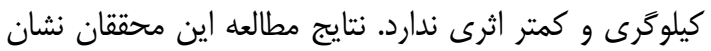

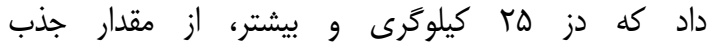
ايمونو كلوبولينها مى كاهد.

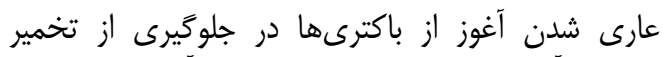

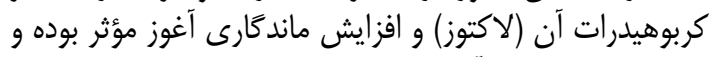

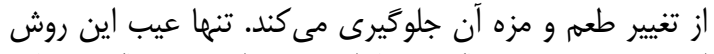

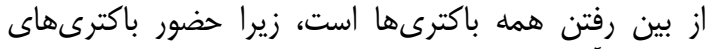

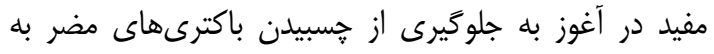

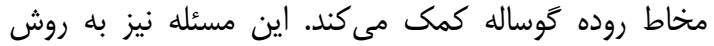

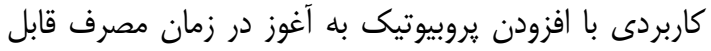

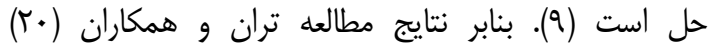

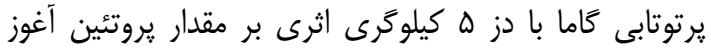

$$
\text { نداشت. }
$$

\section{الكَى زيرواحدهاى ثيروتئين آغوز}

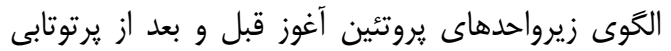

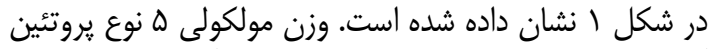

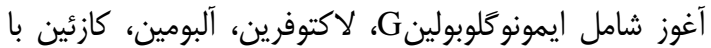

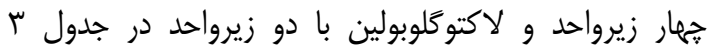

جدول س- زيرواحدهاى يروتئين آغوز و وزن مولكولى آنها (كيلو دالتون) Table 3. Colostrum protein subunits and their molecular weights (kDa)

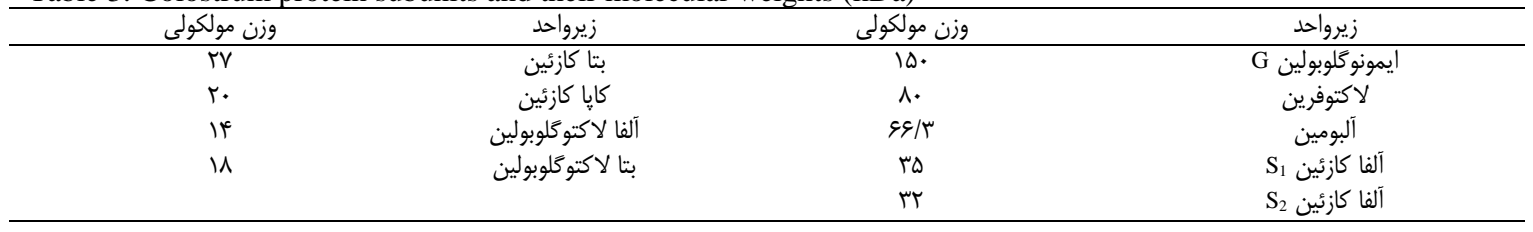


r. استفاده از برتوتابى كاما براى حذف آلودگى باكتريايى آغوز كاو.

دز لازم براى كاهش يك سيكل لكاريتمى از جمعيت إنمايت

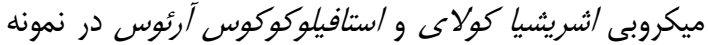

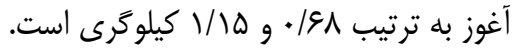

باتوجه به نتايج اين يزوهش مى توان از دزهاى پإيين يرتو

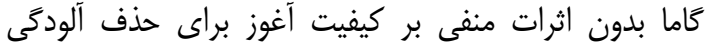

اشريشيا كولاى و استافيلوكوكوس آرئوس استفاده كرد. immunoglobulin G concentration in colostrums. Proceeding of National Congress on Animal and Poultry in North of Iran. Sari Agricultural Science and Natural Resources University, pp: 1614-1617 (In Persian).

2. Amini-robati, E., P. Shawrang, F. Fattahnia and A. Mehrabi. 2014. The comparison between irradiation and freezing effects on colostrum quality. The $6^{\text {th }}$ Iranian Congress on Animal Science. The University of Tabriz, $113 \mathrm{pp}$ (In Persian).

3. Association of Official Analytical Chemists. 2000. Official Methods of Analysis of the Association of Official Analytical Chemists, 17th edition Arlington, VA.

4. Bradford, M.M. 1997. A rapid and sensitive method for the quantitation of microgram quantities of protein utilizing the principle of protein-dye binding. Analytical Biochemistry, 72: 248.

5. Campbell, J.M., L.E. Russell, J.D. Crenshaw, E.M. Weaver, S. Godden, J.D. Quigley, J. Coverdale and H. Tyler. 2007. Impact of irradiation and immunoglobulin $G$ concentration on absorption of protein and immunoglobulin $\mathrm{G}$ in calves fed colostrum replacer. Journal of Dairy Science, 90: 57265731.

6. Esterbauer, H. and K.H. Cheeseman. 1990. Determination of aldehydic lipid peroxidation products: malonaldehyde and 4-hydroxynonenal. Methods in Enzymology, 186: 407-421.

7. Food and Agriculture Organization. 2003. General Standard for Irradiated Foods, CODEX STAN1061983, Rev.1-2003, FAO/WHO, Rome.

8. Garbarino, C., G. Cammi, I. Archetti, M. Amadori, G. Panella, M. Ricchi, V. Tranquillo and N. Arrigoni. 2012. Heat treatment and gamma-irradiation of bovine colostrum: Impact on survival of Mycobacterium avium subsp. paratuberculosis (MAP) and other pathogens and on immunoglobulin content Proceedings of the 11th International Colloquium on Paratuberculosis. Sydney, Australia, 197.

9. Garin-Bastuji, B., B. Perrin, M.F. Thorel and J.L. Martel. 1990. Evaluation of $\gamma$-ray irradiation of cows' colostrum for Brucella abortus, Escherichia coli K99, Salmonella dublin and Mycobacterium paratuberculosis decontamination. Letters in Applied Microbiology, 11: 163-16.

10. Green, L., S. Godden and J. Feirtag. 2003. Effect of batch and high temperature-short time pasteurization on immunoglobulin G concentrations in colostrum. Journal of Dairy Science, 86246 pp.

11. Godden, S.M., S. Smith, J.M. Feirtag, L.R. Green, S.J. Wells and J.P. Fetrow. 2003. Effect of OnFarm Commercial Batch Pasteurization of Colostrum on Colostrum and Serum Immunoglobulin Concentrations in Dairy Calves. Journal of Dairy Science, 86: 1503-1512.

12. Godden, S., S. McMartin, J. Feirtag, J. Stabel, R. Bey, S. Goyal, L. Metzger, J. Fetrow, S. Wells and H. Chester-Jones. 2006. Heat-Treatment of Bovine Colostrum. II: Effects of Heating Duration on Pathogen Viability and Immunoglobulin G. Journal of Dairy Science, 89: 3476-3483.

13. Laemmli, U.K. 1970. Cleavage of structural proteins during the assembly of the head of bacteriophage T4. Nature, 227: $680 \mathrm{pp}$.

14. McGuirk, S. and M. Collins. 2004. Managing the production, storage and delivery of colostrum. in Veterinary Clinics of North America Food Animal Practice. W.B. Saunders, New York, NY, 593-603.

15. McMartin, S., S. Godden, L. Metzger, J. Feirtag, R. Bey, J. Stabel, S. Goyal, J. Fetrow, S. Wells and H. Chester-Jones. 2006. Heattreatment of bovine colostrums. I: Effects of temperature on viscosity and immunoglobulin G level. Journal of Dairy Science, 89: 2110-2118.

16. Negrulescu, A., V. Patrulea, M.M. Mincea, C. Ionascu, B.A. Vlad-Oros and V. Ostafe. 2012. Adapting the reducing sugars method with dinitrosalicylic acid to microtiter plates and microwave heating. Journal of the Brazilian Chemical Society, 23: 2176-2182.

17. Poulsen, K.P., F.A. Hartmann and S.M. McGuirk. 2002. Bacteria in colostrum: impact on calf health, in Proc. 20th American College of Internal Veterinary Medicine: Mira Digital Publishing. 773 pp.

18. Shannon, L.H., C. Vargas-Aburto, M.U. Roberto and C.J. Woolverton. 2005. Inactivation of Bacillus Endospores in envelopes by electron beam irradiation. Applied and Environmental Microbiology, 1171.

19. Trampuz, A., K.E. Piper, J.M. Steckelberg and R. Patel. 2006. Effect of gamma irradiation on viability and DNA of Staphylococcus epidermidis and Escherichia coli. Journal of Medical Microbiology, 55: 1271-1275.

20. Tran, H., K. Marlowe, K. McKenney, G. Petrosian, Y. Griko, W.H. Burgess, W.N. Drohan, MA. Imboden, C. Kempf, N. Boschetti and D.M. Mann. 2004. Functional integrity of intravenous immunoglobulin following irradiation with a virucidal dose of gamma radiation. Biologicals, 32: 94104. 


\title{
Application of Gamma Irradiation for Eliminating Bacterial Contamination of Bovine Colostrums
}

\author{
Parvin Shawrang ${ }^{1}$, Mohsen Mortaheb ${ }^{2}$, Mehdi Behgar ${ }^{3}$, Farahnaz Motamedi-sede $^{3}$ and \\ Hamed Askari ${ }^{4}$
}
1- Assistant Professor, Nuclear Agriculture Research School, Nuclear Science and Technology Research Institute, Atomic Energy Organization of Iran (Corresponding Author: pshawrang@ nrcam.org) 2- Experts, Fajr-Isfahan Agricultural Co
3 and 4- Assistant Professor and Expert, Nuclear Agriculture Research School, Nuclear Science and Technology Research Institute, Atomic Energy Organization of Iran Received: February 8, 2017 Accepted: November 6, 2017

\begin{abstract}
In order to colostrum pasteurization and creating of dose-response curve for Escherichia coli and Staphylococcus aurous, the colostrum of cow was gamma irradiated at doses of $0,0.5,1$ and 2 KGy. Chemical composition, true protein, electrophoretic pattern of proteins, lactose content, peroxide level and $\mathrm{pH}$ of colostrums samples before and after of irradiation were determinate. The obtained data were analysed using proc GLM of SAS software appropriate for completely randomized design. The effect of irradiation was significant on Escherichia coli and Staphylococcus aurous contamination of colostrums $(\mathrm{p}<0.05)$. The Escherichia coli and Staphylococcus aurous contamination decreased as irradiation dose increased and reach to zero at dose of $2 \mathrm{kGy}$. Gamma irradiation had no effect ( $\mathrm{p}>0.05)$ on chemical composition, true protein, fatty acid composition, peroxide level, $\mathrm{pH}$ and electrophoretic pattern of proteins and lactose content. Based on the results of this study, the appropriate dose for decrease in log cycle of Escherichia coli and Staphylococcus aurous population in colostrum is 0.68 and $1.15 \mathrm{kGy}$ respectively. Therefore, gamma irradiation at low doses can eliminate Escherichia coli and Staphylococcus aurous contamination without negative impact on colostrum quality.
\end{abstract}

Keywords: Colostrum, Chemical composition, Escherichia coli, Gamma irradiation, Staphylococcus aurous, 